\title{
Contrasting Efforts: The Microbiome and Type 1 Diabetes
}

\author{
Jordan T Russell and Eric W Triplett* \\ Department of Microbiology and Cell Science, Institute of Food and Agricultural Sciences, University of Florida, Gainesville, FL, USA
}

\begin{abstract}
In the search for environmental triggers of type 1 diabetes (T1D), disease associations with the human gut microbiome are of great interest. Three studies of children at high genetic risk for T1D have examined associations between microbial taxa and the development of T1D autoimmunity: DIPP (Diabetes Prediction and Prevention), BABYDIET (Primary Prevention of Type 1 Diabetes in Relatives at Increased Genetic Risk), and DIABIMMUNE (Pathogenesis of Type 1 Diabetes- Testing the Hygiene Hypothesis). These studies are herein examined for their findings, including areas for improvement and means to go beyond preliminary taxonomic associations for predicting functional influences by microbes. These studies serve as useful tools for guiding future research and deserve careful consideration.
\end{abstract}

Keywords: Bacteroides dorei; Network associations; Multi-omics; Geography

\section{Introduction}

The increasing incidence of autoimmune type 1 diabetes (T1D) in developed countries is known to have surpassed levels attributable solely to genetic susceptibility, suggesting an environmental component to the disease as a likely contributing factor [1]. This environmental component could manifest as a trigger for autoimmune progression or perhaps a loss in a protective function. Many hypotheses have been proposed to explain the increase in T1D incidence but no single cause seems to fit every scenario [2]. The hygiene hypothesis posits that lack of early childhood infections, or rather exposure to infectious agents at a young age, impedes proper immune system development, and that this phenomenon occurs because of increased sanitary living conditions in the developed world [3]. This however contradicts findings that viral infection may act as the trigger for T1D, although other studies demonstrate that viral infection in some cases may have a protective function, and in some instances viral exposure has decreased in areas where T1D prevalence is highest [4-7]. Furthermore, other factors including vitamin $\mathrm{D}$ and diet may play a more widespread or influential role in T1D, although some studies have ruled no association to vitamin $\mathrm{D}$ and T1D autoimmunity $[1,8]$. When executing a study aimed at associating changes in the microbiome with disease, a carefully designed, holistic approach is needed and the effects of potential confounders must be considered.

\section{Literature Review}

\section{Diabetes prediction and prevention}

The earliest work with a human cohort on connections between the microbiome and T1D was done with samples from the Diabetes Prediction and Prevention (DIPP) study in Finland [9-12]. In the beginning, this study was carried out with very few samples but hinted at both taxonomic and functional differences between children with and without T1D $[9,10]$. Later work with many more subjects from Turku, Finland showed a significantly higher number and relative abundance of Bacteroides dorei in autoimmune subjects compared to healthy subjects several months prior to autoimmunity [13]. During that time, 947 stool samples were collected at monthly intervals from at-risk children between 4 months and 2 years of age, representing 47 healthy controls and 29 children who were positive for at least two autoantibodies, 22 of which later developed T1D (cases). This analysis also demonstrated no connection between antibiotic use and future T1D autoimmunity in keeping with the observation that Bacteroides dorei genomes showed a high level of antibiotic resistance [13]. These efforts, particularly the metagenomics done by Brown et al. (2011), led to the hypothesis that butyrate production by gut bacteria may prevent T1D in children $[9,14]$.

\section{Pathogenesis of type 1 diabetes- Testing the hygiene hypothesis (DIABIMMUNE)}

The DIABIMMUNE study has two aims: 1 ) determine the role the hygiene hypothesis plays in T1D and other autoimmune diseases and 2) define the mechanisms that lead to immune dysregulation based on the findings of aim $1[15,16]$. This is a relatively large, longitudinal study that enrolled 387, 330, and 115 babies followed prospectively from birth to 3 years of age in Finland, Estonia and Karelian Russia, respectively.

Kostic et al. published results on an analysis of the microbiome of DIABIMMUNE study subjects from Finland and Estonia [17]. In total, 33 infants genetically predisposed to T1D were sampled at time points between birth and 3 years old. Of these subjects, 22 were matched healthy controls, 7 were subjects who seroconverted (seroconverters) and 4 were subjects who seroconverted and later developed T1D (cases). It is important to note that only six subjects were from Estonia including four controls, one autoimmune subject, and one with T1D, while the remaining 27 subjects were from Finland.

A plateau in alpha diversity in T1D subjects was described when autoimmune and healthy subjects experienced increasing gut microbial diversity. This occurred in the time window between seroconversion and T1D diagnosis. However, despite almost monthly sampling from seroconverters and controls, only one case subject was sampled consistently in this time frame, making this conclusion based on a single subject. Furthermore, no associations of specific bacterial taxa and T1D outcome were described, perhaps owing to small sample size. This highlights an important issue regarding consistent sampling

*Corresponding author: Eric W. Triplett, Department of Microbiology and Cell Science, Institute of Food and Agricultural Sciences, University of Florida, 1355 Museum Rd., P.O. Box 110700, Gainesville, FL, 32611-0700, USA, Tel: 1-352-392 1906; E-mail: ewt@ufl.edu

Received: September 15, 2017; Accepted: October 03, 2017; Published October 07, 2017

Citation: Russell JT, Triplett EW (2017) Contrasting Efforts: The Microbiome and Type 1 Diabetes. Diabetes Case Rep 2: 129. doi: 10.4172/2572-5629.1000129

Copyright: @ 2017 Russell JT, et al. This is an open-access article distributed under the terms of the Creative Commons Attribution License, which permits unrestricted use, distribution, and reproduction in any medium, provided the original author and source are credited. 
among a large pool of subjects for each outcome category that should be observed in any microbiome-related study aiming to make statistically significant comparisons. In addition, confounders such as antibiotic use in these subjects could not be accounted for due to a lack of properly annotated metadata and was therefore assumed to have a fixed effect. The importance of extensive and well documented metadata should be underscored for any future microbiome analysis to remove the possibility of confounding data. Additionally, Vatenan et al. proposed a role for differences in lipopolysaccharide (LPS) contribution based on differentially abundant bacteria in the DIABIMMUNE study, however the analysis on LPS structure was done on isolates not associated with disease and with isolates not collected from subjects in the DIABIMMUNE study [18]. Thus, further work is necessary.

\section{Primary prevention of type 1 diabetes in relatives at increased genetic risk (BABYDIET)}

A comparison of the work from DIPP and BABYDIET illustrates the importance of the confounder of geography when attempting to make bacterial associations with disease. As described above, the DIPP study found a strong bacterial association with T1D autoimmunity. Although no such associations were found analyzing the gut bacterial communities of 298 stool samples from 44 subjects in the German BABYDIET study, a substantial decline in microbial interaction networks were observed in subjects who developed islet autoimmunity versus those who did not [19]. Further analyses showed that significant bacterial taxonomic differences were associated with islet autoimmunity in those subjects with a complex food diet early in life [20]. Bacteroides was high in subjects who became autoimmune for T1D while Akkermansia was higher in subjects who remained healthy.

\section{Discussion}

\section{Comparing the cohorts}

A consensus is building behind the notion that bacteria in the genus Bacteroides are in high numbers some months prior to islet autoimmunity but remain low in abundance in healthy children. This was observed in DIPP where $B$. dorei had a higher relative and absolute abundance of this species approximately 8 months prior to seroconversion [13] and in BABYDIET in those children provided a complex diet early in life [20]. In DIABIMMUNE, an intriguing hypothesis was raised that the LPS structure of $B$. dorei may prevent immunotolerance in the gut thereby leading to autoimmunity [18]. However, this idea requires more testing with the appropriate genetic constructs of $B$. dorei. However, few bacteria have been strongly associated with health in these cohorts apart from Akkermansia in BABYDIET [20]. Bacteria associated with health in human cohorts may prove to be useful as probiotics for the prevention of T1D. Such bacteria need to be isolated and characterized to discern mechanisms that may be associated with health in animal models. Work from the TEDDY (The Environmental Determinants of Diabetes in the Young) study showed the great variation in gut microbiome composition among the six clinical sites used in TEDDY [21]. This suggests that bacteria associated with health may be difficult to identify by their taxonomy since the bacterial species are not likely to be sampled around the world.

\section{Conclusion}

Comparing results from multiple cohorts can reveal important trends and suggest directions for future work. More integrated, systems approaches are needed to assess the roles of gut bacteria and viruses in the development of pediatric T1D. The role of phage and bacterial epigenetics also needs to be explored in the future [22]. Meta-omics such as meta transcriptomics and meta proteomics can provide a more comprehensive and quantitative view of which genes and proteins are being expressed and produced [23]. Large metabolomics studies are also warranted in the future. This can complement metagenomics which can only provide functional potential. The value of a holistic multi-omic approach has been stated previously and this practice has been adopted in recent gut microbiome studies [1,21,24]. Great effort has recently been invested in identifying environmental contributors to T1D autoimmunity originating in the human gut. Complex, longitudinal studies such as DIPP and DIABIMMUNE have proved useful in providing direction for future research aimed at functional hypotheses between gut microbes and T1D. Vatenan et al. proposed a role for differences in lipopolysaccharide (LPS) contribution based on differentially abundant bacteria in the DIABIMMUNE study, however the analysis on LPS structure was done on isolates not associated with disease and further work is necessary [18]. Additional efforts from these studies have also lead to connections between T1D and the intestinal virome/epigenome that are worthy of further investigation $[22,25]$. These studies highlight the importance of careful study design and a holistic mult-omic approach to better identify all the functional changes in the gut as it relates to disease.

\section{Conflict of Interest}

The authors declare no conflict of interest in the submission of this manuscript

\section{References}

1. Atkinson MA, Eisenbarth GS, Michels AW (2014) Type 1 diabetes. Lancet 383 : 69-82.

2. Egro FM (2013) Why is type 1 diabetes increasing? J Mol Endocrinol 51: R1-13

3. Bach JF, Chatenoud L (2012) The hygiene hypothesis: An explanation for the increased frequency of insulin-dependent diabetes. Cold Spring Harb Perspect Med 2: a007799.

4. Coppieters KT, Boettler T, Von Herrath M (2012) Virus infections in type 1 diabetes. Cold Spring Harb Perspect Med 2: a007682.

5. Yeung WC, Rawlinson WD, Craig ME (2011) Enterovirus infection and type 1 diabetes mellitus: Systematic review and meta-analysis of observational molecular studies. BMJ 342: d35

6. Graves PM, Rotbart HA, Nix WA, Pallansch MA, Erlich HA, et al. (2003) Prospective study of enteroviral infections and development of beta-cell autoimmunity. Diabetes autoimmunity study in the young (DAISY). Diabetes Res Clin Pract 59: 51-61

7. Viskari H, Ludvigsson J, Uibo R, Salur L, Marciulionyte D, et al. (2005) Relationship between the incidence of type 1 diabetes and maternal enterovirus antibodies: time trends and geographical variation. Diabetologia 48: 1280-1287.

8. Reinert-Hartwall L, Honkanen J, Härkönen T, llonen J, Simell O, et al. (2014) No association between vitamin $D$ and $\beta$-cell autoimmunity in Finnish and Estonian children. Diabetes Metab Res Rev 30: 749-760.

9. Brown CB, Davis-Richardson AG, Giongo A, Gano KA, Crabb DB, et al. (2011) Gut microbiome metagenomics analysis suggests a functional model for the development of autoimmunity for type 1 diabetes. PLoS ONE 6: e25792.

10. Giongo A, Mukherjee N, Gano KA, Crabb DB, Casella G, et al. (2011) Toward defining the autoimmune microbiome for type 1 diabetes. Isme J 5: 82-91.

11. Lönnrot M, Korpela K, Knip M, Ilonen J, Simell O, et al. (2000) Enterovirus infection as a risk factor for beta-cell autoimmunity in a prospectively observed birth cohort: The Finnish Diabetes Prediction and Prevention Study. Diabetes 49: 1314-1318.

12. Nejentsev S, Sjöroos M, Soukka T, Knip M, Simell O, et al. (1999) Populationbased genetic screening for the estimation of Type 1 diabetes mellitus risk in Finland: Selective genotyping of markers in the HLA-DQB1, HLA-DQA1 and HLA-DRB1 loci. Diabet Med 16: 985-992.

13. Davis-Richardson AG, Ardissone AN, Dias R, Simell V, Leonard MT, et al. (2014) Bacteroides dorei dominates gut microbiome prior to autoimmunity in Finnish children at high risk for type 1 diabetes. Front Microbiol 5: 678. 
Citation: Russell JT, Triplett EW (2017) Contrasting Efforts: The Microbiome and Type 1 Diabetes. Diabetes Case Rep 2: 129. doi: 10.4172/25725629.1000129

14. Davis-Richardson AG, Triplett EW (2015) A model for the role of gut bacteria in the development of autoimmunity for type 1 diabetes. Diabetologia 58: 13861393.

15. Peet A, Kool P, llonen J, Knip M, Tillmann V, et al. (2012) Birth weight in newborn infants with different diabetes-associated HLA genotypes in three neighbouring countries: Finland, Estonia and Russian Karelia. Diabetes Metab Res Rev 28: 455-461.

16. Peet A, Hämäläinen AM, Kool P, llonen J, Knip M, et al. (2014) Early postnata growth in children with HLA-conferred susceptibility to type 1 diabetes. Diabetes Metab Res Rev 30: 60-68.

17. Kostic AD, Gevers D, Siljander H, Vatanen T, Hyötyläinen T, et al. (2015) The dynamics of the human infant gut microbiome in development and in progression toward type 1 diabetes. Cell Host Microbe 17: 260-273.

18. Vatanen T, Kostic AD, d'Hennezel E, Siljander H, Franzosa EA, et al. (2016) Variation in microbiome LPS immunogenicity contributes to autoimmunity in humans. Cell 165: 1551.

19. Endesfelder D, Zu Castell W, Ardissone A, Davis-Richardson AG, Achenbach $P$, et al. (2014) Compromised fecal microbiota networks in children with islet autoimmunity. Diabetes 63: 2006-2014.
20. Endesfelder D, Engel M, Davis-Richardson AG, Ardissone AN, Achenbach $P$ et al. (2016) Towards a functional hypothesis relating anti-islet autoimmunity to the dietary impact on microbial communities and butyrate production. Microbiome 4: 17.

21. Kemppainen KM, Ardissone AN, Davis-Richardson AG, Fagen JR, Gano KA, et al. (2015) Early childhood gut microbiomes show strong geographic differences among subjects at high risk for type 1 diabetes. Diabetes Care 38: 329-332.

22. Leonard MT, Davis-Richardson AG, Ardissone AN, Kemppainen KM, Drew JC et al. (2014) The methylome of the gut microbiome: Disparate Dam methylation patterns in intestinal Bacteroides dorei. Front Microbiol 5: 361.

23. Franzosa EA, Hsu T, Sirota-Madi A, Shafquat A, Abu-Ali G, et al. (2015) Sequencing and beyond: Integrating molecular 'omics' for microbial community profiling. Nat Rev Microbiol 13: 360-372.

24. Heintz Buschart A, May P, Laczny CC, Lebrun LA, Bellora C, et al. (2016) Integrated multi-omics of the human gut microbiome in a case study of familial type 1 diabetes. Nat Microbiol 2: 16180.

25. Zhao G, Vatenan T, Droit L, Park A, Kostic AD, et al. (2017) Instestinal virome changes precede autoimmunity in type 1 diabetes-susceptible children. Proc Natl Acad Sci USA 114: E6166-E6175. 